\title{
P22 Central and Peripheral Contributions to Submaximal Exercise Performance in Older Adults in the SouthAll and Brent REvisited (SABRE) Study
}

\author{
Siana Jones ${ }^{1, *}$, Martin Schultz ${ }^{2}$, Therese Tillin ${ }^{1}$, Suzanne Williams ${ }^{1}$, Nishi Chaturvedi ${ }^{1}$, Alun Hughes ${ }^{1}$ \\ ${ }^{1}$ University College London, London, UK \\ ${ }^{2}$ Menzies Institute for Medical Research, University of Tasmania, Australia
}

\begin{abstract}
Background: Functional capacity declines with age leading to disability and increased cost of health and social care. In older adults, factors that influence the ability to sustain sub-maximal exercise (a marker of functional capacity) are not well defined. We aimed to determine the contribution of cardiac function, skeletal muscle oxygen desaturation, extraction and oxidative capacity to sub-maximal exercise performance in a population-based sample of older adults.

Methods: Participants in the SABRE study undertook a 6-minute stepper test (6MST). Sub-maximal exercise performance was assessed as the highest achieved whole-body oxygen uptake $\left(\mathrm{VO}_{2}\right)$ during the $6 \mathrm{MST}$. Near Infrared Spectroscopy (NIRS) was used to locally assess skeletal muscle oxygen extraction, desaturation and maximum oxidative capacity. Echocardiography was used to measure cardiac function. Analysis was by multiple linear regression adjusted for confounders: age, sex, ethnicity, diabetes and obesity.
\end{abstract}

Results: 395 participants $(73.0 \pm 6.3$ years old, male; $n=229)$ undertook the 6MST, skeletal muscle desaturation measurements and echocardiography. Skeletal muscle desaturation and cardiac output were independently associated with whole-body $\mathrm{VO}_{2}$ (standardized $\beta$ : $-0.20, p<0.001 \& 0.47, p<0.001$, respectively). Local skeletal muscle oxygen extraction was independently associated with whole-body $\mathrm{VO}_{2}$ (standardized $\beta$ adjusted for confounders \& cardiac output: $0.32, p<0.001$ ). Maximum oxidative capacity was not associated with $\mathrm{VO}_{2}(\beta(95 \%$ CI: $0.02(-0.01,0.04), p=0.152)$.

Conclusion: Local oxygen extraction and desaturation in skeletal muscle are independent predictors of sub-maximal exercise performance in older adults. These associations persist with adjustment for cardiac output suggesting local capacity to extract oxygen could be a major limiting factor functional capacity in older adults.

(C) 2019 Association for Research into Arterial Structure and Physiology. Publishing services by Atlantis Press International B.V. This is an open access article distributed under the CC BY-NC 4.0 license (http://creativecommons.org/licenses/by-nc/4.0/). 